\title{
Variabilités bioclimatiques et distribution spatiale des herbacées fourragères dans le Moyen-Bénin (Afrique de l'Ouest)
}

\author{
A. R. A. SALIOU ${ }^{1,2^{*}}$, M. OUMOROU ${ }^{1}$ et A. B. SINSIN $^{2}$ \\ ${ }^{1}$ Laboratoire de Recherche en Biologie Appliquée (LaRBA/EPAC/UAC), 01 BP 2009, Cotonou, Bénin. \\ ${ }^{2}$ Laboratoire d'Ecologie Appliquée (LEA/FSA/UAC), 02 BP 388 Parakou, Bénin. \\ *Auteur correspondant ; E-mail : salitour2005@yahoo.fr; Tel : (00229) 97874791.
}

\section{RESUME}

Les menaces continues des parcours de transhumance sont dues aux variabilités des facteurs climatiques accentuées par les activités anthropiques. La dégradation de ces écosystèmes en zone soudanoguinéenne a des conséquences sur la disponibilité de fourrage pour l'alimentation du bétail. L'étude sur la compréhension de la distribution géographique des herbacées fourragères les plus appétées au cours des périodes de transhumance de bétail dans le Moyen-Bénin, a été possible grâce à la modélisation à partir de l'algorithme de Maximum Entropie. Le présent article se propose d'une part de déterminer les étendues des habitats potentiels présents et de prédire la distribution future de ces herbacées fourragères dans le MoyenBénin. A cet effet, 988 points d'observations dont (184) pour Andropogon chinensis, (350) pour Andropogon gayanus, (127) pour Andropogon schirensis et (327) pour Andropogon tectorum ont été utilisés pour la modélisation. Le modèle MIROC avec deux scénarii d'émission de Gaz à Effet de Serre dont l'un pessimiste RCP 2.6, et l'autre optimiste RCP 8.5 a contribué à faire tourner 19 variables bioclimatiques et 3 variables environnementales. Les résultats obtenus ont permis de déterminer les habitats potentiels des différentes herbacées fourragères et d'établir une corrélation entre leur distribution et l'évolution des paramètres environnementaux et bioclimatiques.

(C) 2014 International Formulae Group. All rights reserved.

Mots clés : Distribution géographique, variabilités climatiques, herbacées fourragères, Moyen-Bénin.

\section{INTRODUCTION}

Les processus naturels jouent un rôle déterminant dans le fonctionnement et la dynamique des systèmes écologiques (Rodiguez et al., 2007). La productivité des écosystèmes est étroitement dépendante de la pluviosité, de la texture et de la profondeur du sol (Zékpété, 2009). La biomasse herbacée varie dans le temps suivant les conditions pluviométriques et dans l'espace suivant la nature du substrat édaphique liée à la situation topographique (Oumorou, 2003). L'offre fourragère est essentiellement assurée par les parcours naturels (Djenontin, 2005). Ces parcours sont particulièrement soumis à des pressions anthropiques (Houinato et Sinsin, 2000), telles l'agriculture extensive, notamment la production cotonnière, la pratique des feux tardifs de végétation dans le Moyen-Bénin. En Afrique, 25 à $42 \%$ des espèces végétales pourraient être menacées d'extinction du fait d'une perte de 81 à $97 \%$ 
des habitats favorables d'ici 2085 (Parry et al., 2007).

L'utilité de la modélisation dans l'aménagement prospectif des parcours et la gestion des terres a été soulignée par plusieurs auteurs notamment (Peterson, 2007). Les modèles prédictifs sont de plus en plus utilisés pour répondre à ces questions relatives à l'écologie, à la biogéographie et à la distribution des espèces (Rodríguez et al., 2007) par l'identification des grands sites prioritaires. Le présent article met en exergue les relations entre les facteurs bioclimatiques et la distribution spatiale de Andropogon tectorum, Andropogon gayanus, Andropogon chinensis et Andropogon schinensis dans le Moyen-Bénin.

\section{MATERIEL ET METHODES \\ Matériel \\ Milieu d'étude}

La zone de transition soudanoguinéenne située entre la 7è et 9è parallèle Nord avec une superficie de $16900 \mathrm{~km}^{2}$ est le site d'étude (Figure 1).

\section{Données d'occurrence d'espèces}

Les coordonnées géographiques des données de présence des différentes espèces prises à l'aide d'un GPS à chaque $500 \mathrm{~m}$ ont été utilisées. La base de données mise en place a été complétée par les points de présence disponibles sur le site de GBIF (http://www.gbif.org/).

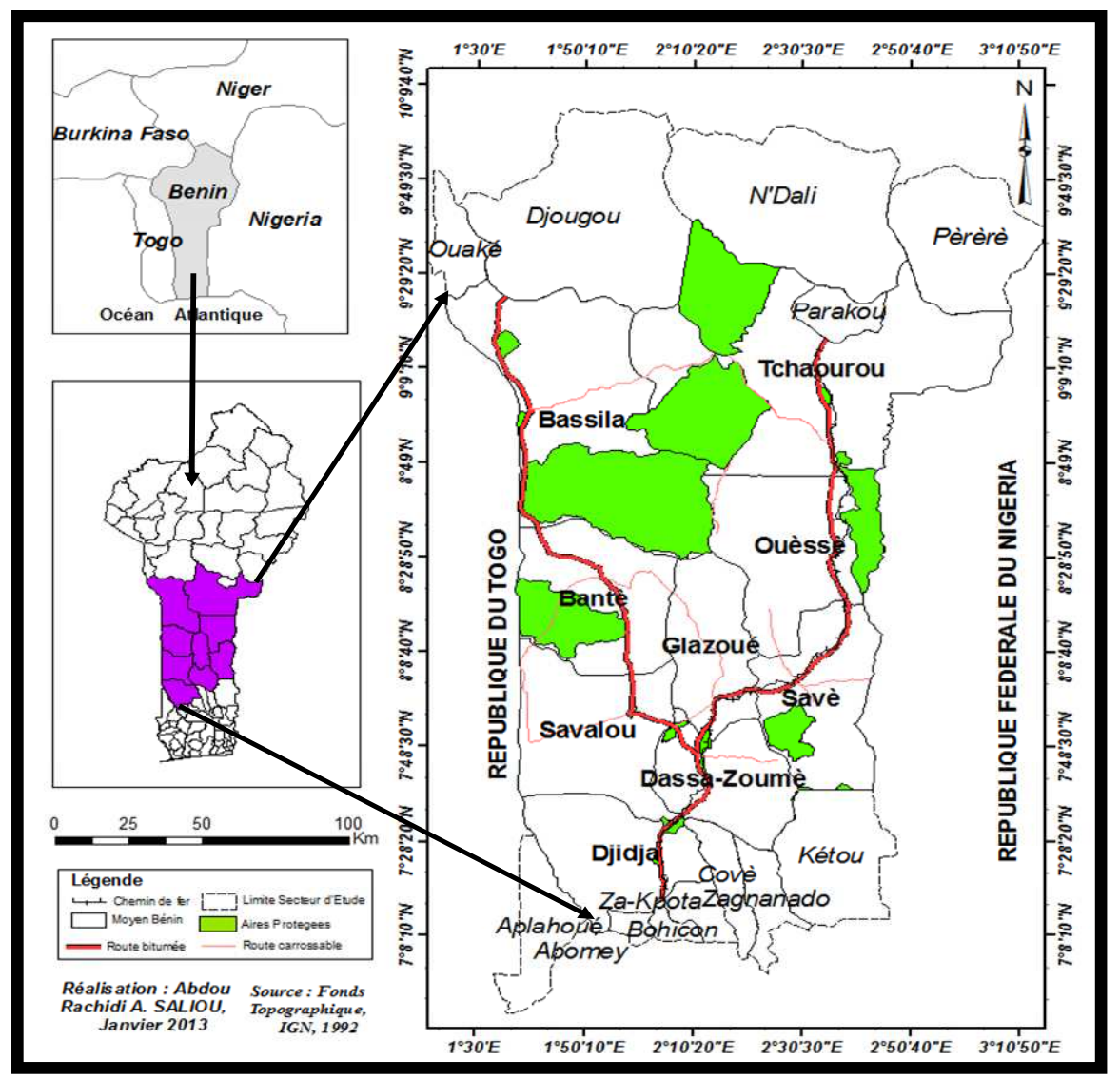

Figure 1 : Carte de situation du Moyen-Bénin. 
Données

bioclimatiques

et environnementales

Sur 22 variables testées, 05 variables bioclimatiques et 02 variables environnementales ont été retenues pour faire tourner le modèle. Il s'agit de : bio4 (Saisonnalité de la température), bio12 (Précipitation annuelle), bio17 (Précipitation du trimestre le plus sec) bio19 (Précipitation du trimestre le plus froid), bio13 (Précipitation $\mathrm{du}$ mois le plus humide), Landcover (Couverture du sol) et Soil (Types de sol).

\section{Méthode de traitement et d'analyse des} données

La méthode du Maximum Entropy (Phillips et al., 2006) a été utilisée pour identifier les facteurs environnementaux déterminant la distribution de Andropogon tectorum, Andropogon gayanus, Andropogon chinensis et Andropogon schinensis. Cette méthode a été choisie parce qu'elle ne requiert pas de données d'absence difficiles à collecter, présente une haute performance et permet d'obtenir une distribution plus proche de la distribution réalisée d'une espèce (Elith et al., 2006). L'idée générale derrière MaxEnt est de trouver une distribution de probabilités, définie sur la zone d'étude, qui satisfait à un ensemble de contraintes dérivées des données d'occurrence de l'espèce. Chaque contrainte exige que la valeur prévue d'une variable environnementale soit dans un intervalle de confiance de sa moyenne empirique (Reed et al., 2008). Pour prédire à l'horizon 2050 la distribution des différentes herbacées fourragères, deux scénarii climatiques ont été utilisés. Il s'agit du scénario optimiste RCP 2.6, et de celui pessimiste RCP 8.0.

\section{RESULTATS}

Le traitement des données d'occurrence des espèces herbacées et des variables bioclimatiques et environnementales dans le modèle Maxent a conduit à la validation du modèle et à la cartographie de la distribution géographique potentielle de ces espèces.

\section{Cartographie de la distribution géographique des herbacées fourragères les plus convoitées par les éleveurs transhumants dans le Moyen-Bénin \\ Deux types de distribution géographique potentielle ont été cartographiés. Il s'agit de la distribution potentielle présente et de la prédiction de la distribution future des herbacées fourragères.}

\section{Distribution présente d'herbacées fourragères dans le Moyen-Bénin}

La distribution des herbacées fourragères les plus appétées dans le MoyenBénin varie suivant les espèces. Ainsi, Andropogon chinensis est très fortement distribuée dans les parties centrale et méridionale, moyennement distribué dans les parties septentrionale et centrale du secteur d'étude (Figure 2a).

En ce qui concerne Andropogon gayanus, il présente un potentiel de distribution très élevé dans la partie septentrionale, moyennement élevé dans la partie centrale et faible dans la partie méridionale du secteur d'étude (Figure 2b).

Pour Andropogon schirensis, elle est très fortement distribuée dans les parties centrale et méridionale, moyennement distribuée dans les parties septentrionale et centrale du secteur d'étude (Figure 2c).

Quant à Andropogon tectorum, elle est très fortement distribuée dans la partie méridionale, moyennement présente dans la partie centrale, avec une probabilité d'aire de distribution très faible dans la partie méridionale (Figure 2d).

Distribution future d'herbacées fourragères dans le Moyen-Bénin

La distribution future des herbacées fourragères à l'horizon 2050 dans le Moyen- 
Bénin varie différemment suivant les scénarii du modèle climatique MIROC utilisés.

Les scénarii RCP 2.6 et RCP 8.5 montrent une prédiction de distribution de Andropogon chinensis avec une probabilité plus élevée au centre, moyenne dans la partie méridionale et faible dans la partie septentrionale de la zone d'étude à l'horizon 2050 (Figures $3 \mathrm{a}$ et 3b).

Le scénario RCP 2.6 prédit une distribution de Andropogon gayanus avec une forte probabilité au sud, une probabilité moyenne de distribution au centre et une faible probabilité au nord du secteur d'étude (Figure 4a). Quant au scénario RCP $8.5 \mathrm{du}$ modèle MIROC, la distribution de l'espèce à l'horizon 2050 est prédit élevée au nord et au sud (Figure 4b).

Le scénario RCP 2.6 montre une prédiction de distribution de Andropogon schirensis à l'horizon 2050, avec une probabilité très élevée du sud au centre et très faible dans la partie septentrionale (Figure 5a). Concernant le scénario RCP 8.5, l'espèce sera distribuée avec une probabilité de distribution plus élevée du sud au centre, et moyenne du centre au nord du secteur d'étude (Figure 5b).

La prédiction de Andropogon tectorum à partir des scénarii climatiques RCP 2.6 et RCP 8.5 du modèle MIROC à l'horizon 2050 traduit une distribution de l'espèce avec une forte probabilité dans la partie centrale, une probabilité moyenne du sud au nord-est, avec une très faible probabilité au nord-ouest du secteur d'étude (Figures 6a et 6b).

Impact des variabilités climatiques sur la distribution des herbacées fourragères les plus convoitées par les transhumants dans le Moyen-Bénin

Les variabilités climatiques ont influencé la distribution présente de Andropogon schirensis, Andropogon gayanus, Andropogon chinensis, et Andropogon tectorum dans le Moyen-Bénin.

La corrélation entre les variables bioclimatiques et la distribution des herbacées fourragères peut s'expliquer à travers la réponse de chaque espèce à l'action de chacune des variables bioclimatiques et environnementales ayant déterminé sa distribution.

Ces courbes montrent comment chaque variable environnementale affecte la prédiction dans Maxent. En effet, la prise en compte de toutes les variables à la fois permet de noter que Andropogon chinensis a répondu favorablement au seuil de probabilité de $100 \%$ à Bio 19 (précipitation du trimestre le plus froid) dans sa contribution à la distribution de l'espèce comparativement à sa réponse défavorable aux variables Bio 13 (Précipitation du mois le plus humide), Bio4 (Saisonnalité de la température), Bio 12 (Précipitations annuelles). On peut donc dire que Bio19 (précipitation du trimestre le plus froid) a été le paramètre le plus déterminant de la distribution géographique potentielle de Andropogon chinensis dans le Moyen-Bénin (Figure 7a).

Sur les six paramètres ayant contribué à la modélisation de Andropogon gayanus, trois ont reçu favorablement la réponse de l'espèce (Figures 8a, 8b, 8c).

Des six variables ayant contribué à la modélisation, seules Bio 12 (Précipitations annuelles) Bio 4 (Saisonnalité de la température), Landcover (couverture du sol) ont reçu favorablement la réponse de Andropogon schirensis (Figures 9a, 9b, 9c). La combinaison de ces trois variables a été plus déterminante dans la distribution de cette espèce.

Par contre, les variables Bio 17 (précipitation du trimestre le plus sec), Bio 13 (Précipitation du mois le plus humide), Bio19 (précipitation du trimestre le plus froid) sont 
défavorables à la réponse de l'espèce (Figures 9d, 9e, 9f).

Les quatre paramètres bioclimatiques auxquelles Andropogon tectorum a répondu favorablement sont Bio 12 (Précipitations annuelles) Bio 4 (Saisonnalité de la température), Bio 17 (précipitation du trimestre le plus sec), Bio19 (précipitation du trimestre le plus froid) (Figures 10a, 10b, 10c, 10d).

Parmi les six variables ayant participé à la modélisation de la distribution de Andropogon tectorum, seules deux ont reçu défavorablement la réponse de l'espèce (Figures 10e, 10f).
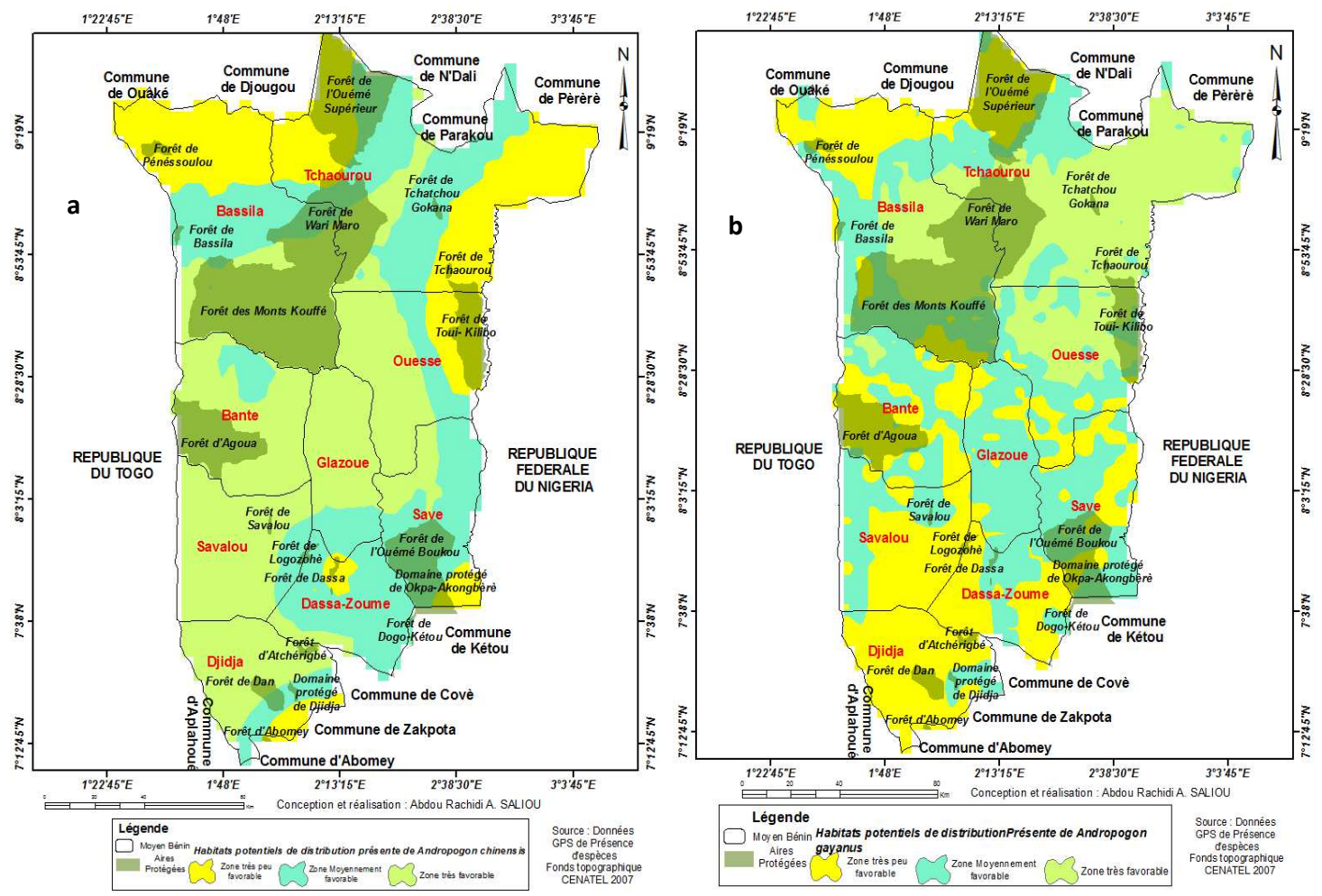

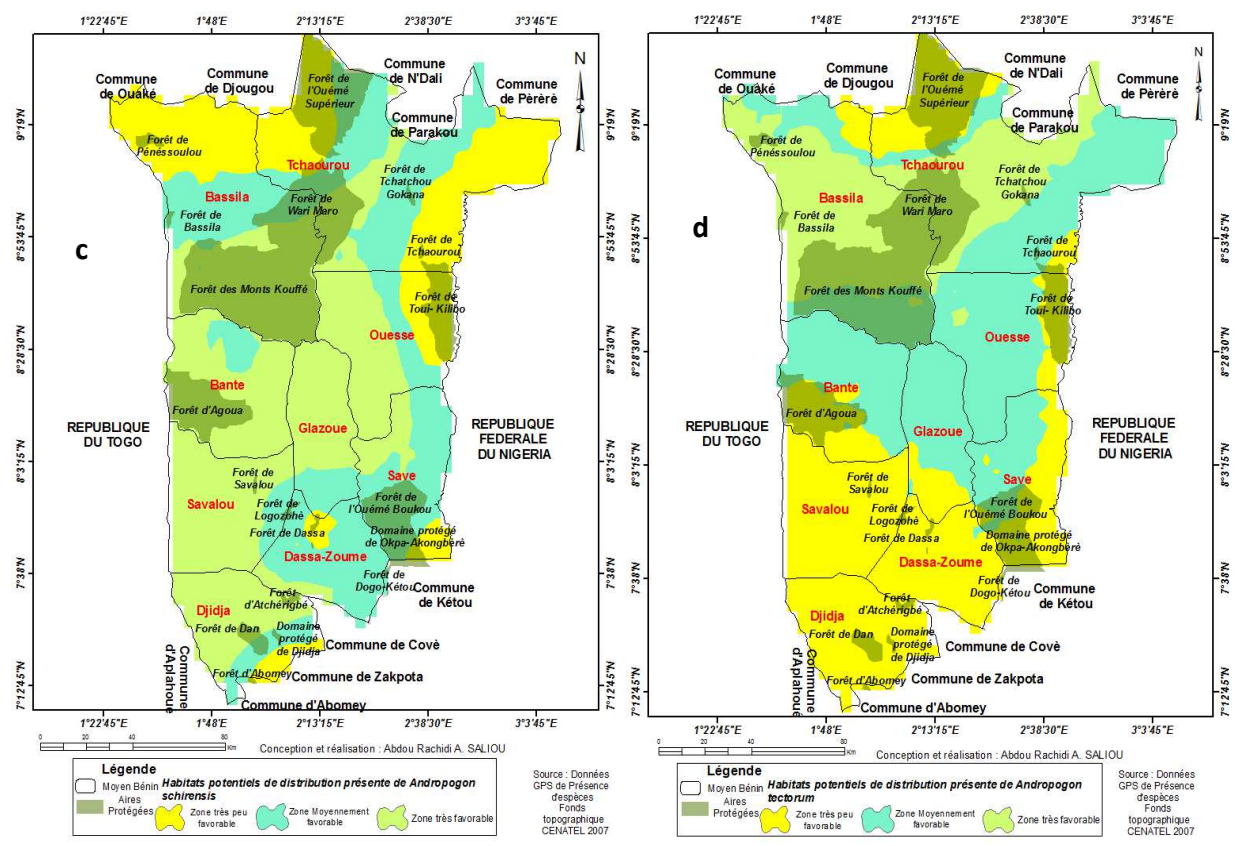

Figures 2 (a,b,c,d): Aires de distribution présente de Andropogon schirensis et Andropogon tectorum dans le Moyen-Bénin.
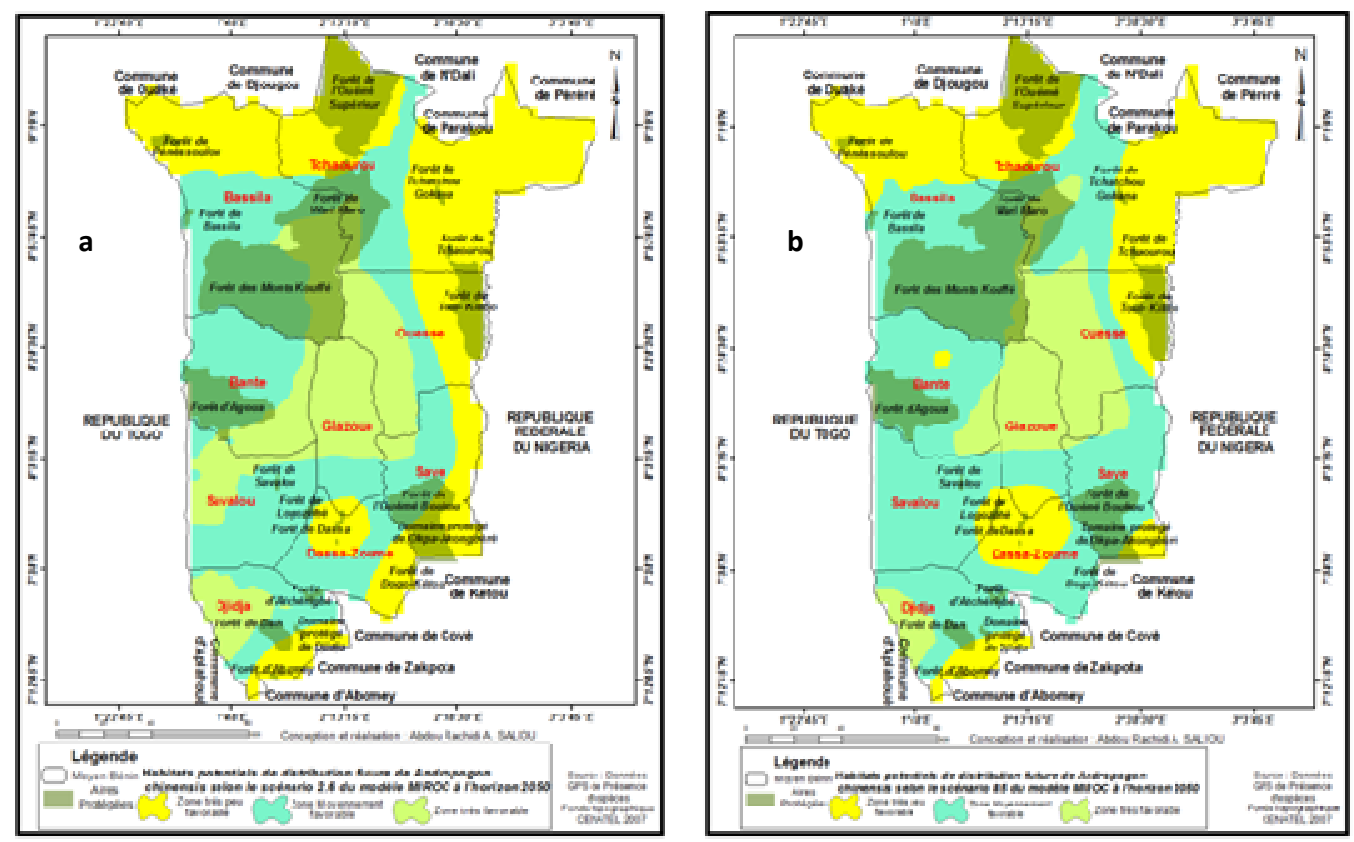

Figures 3 (a,b): Prédictions de distribution de Andropogon chinensis selon les scénarii 2.6 et $8.5 \mathrm{du}$ modèle climatique MIROC à l'horizon 2050 dans le Moyen-Bénin. 

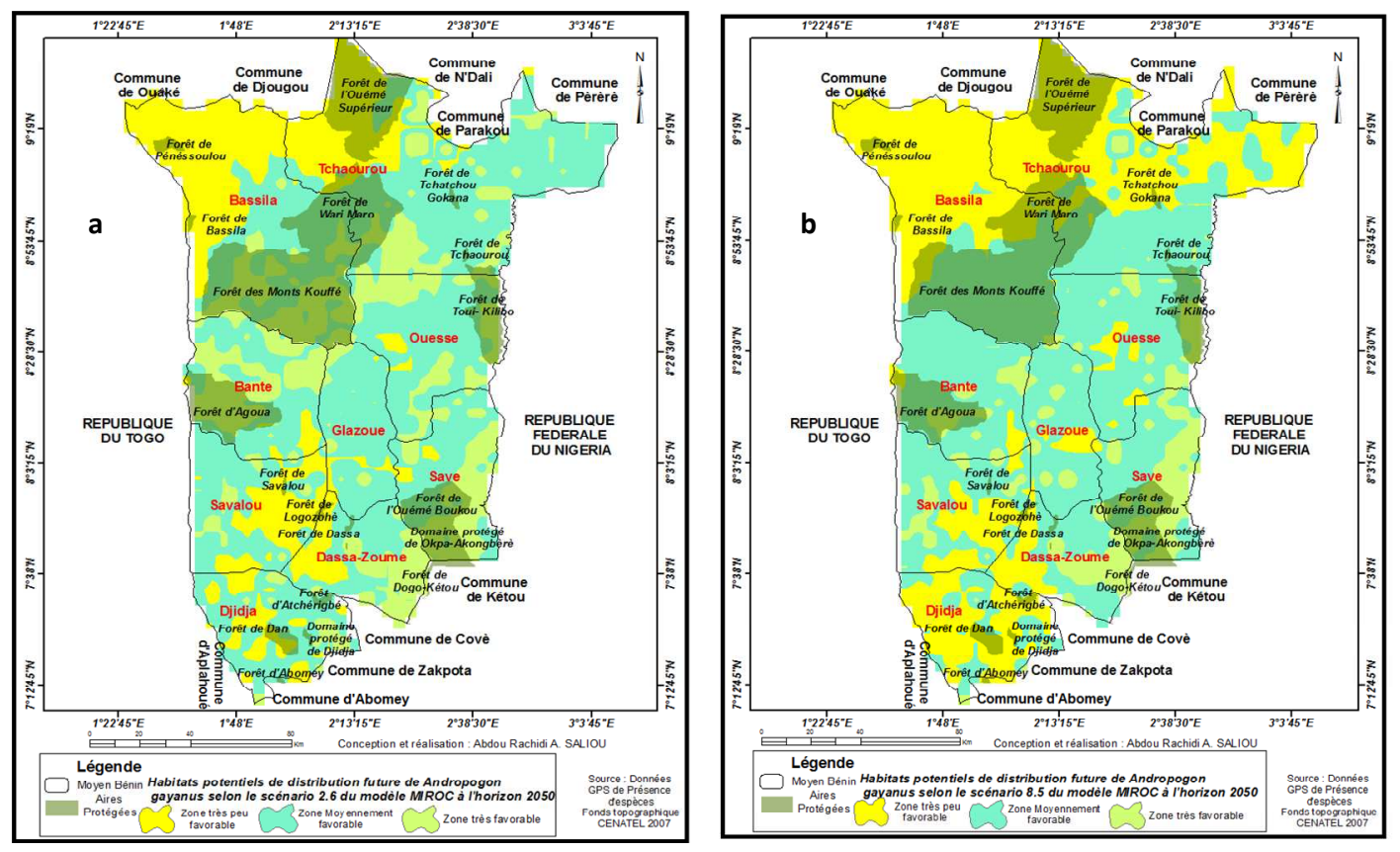

Figures 4 (a,b): Prédictions de distribution de Andropogon gayanus selon les scénarii 2.6 et 8.5 du modèle climatique MIROC à l'horizon 2050 dans le Moyen-Bénin.
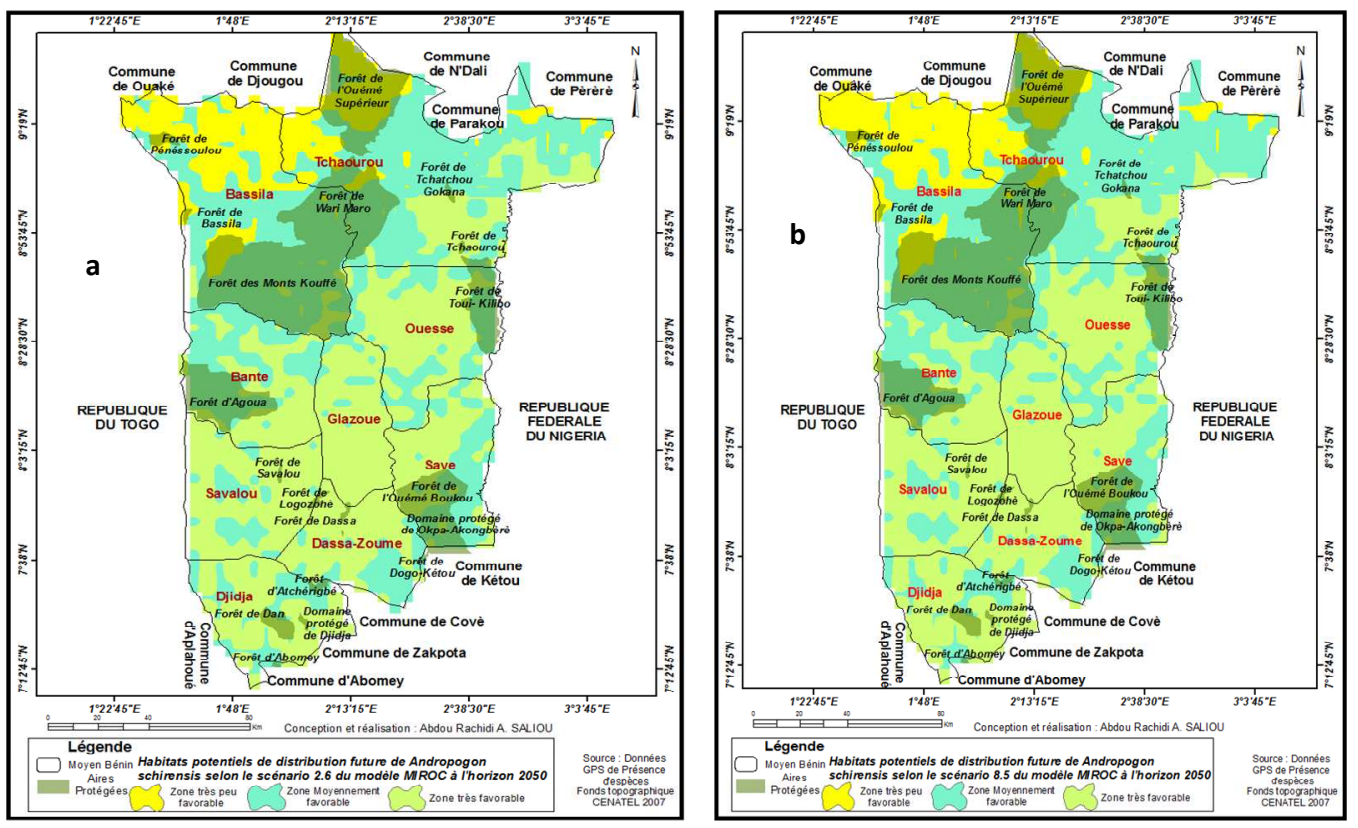

Figures 5 (a,b): Prédictions de distribution de Andropogon schirensis selon les scénarii 2.6 et 8.5 du modèle climatique MIROC à l'horizon 2050 dans le Moyen-Bénin. 

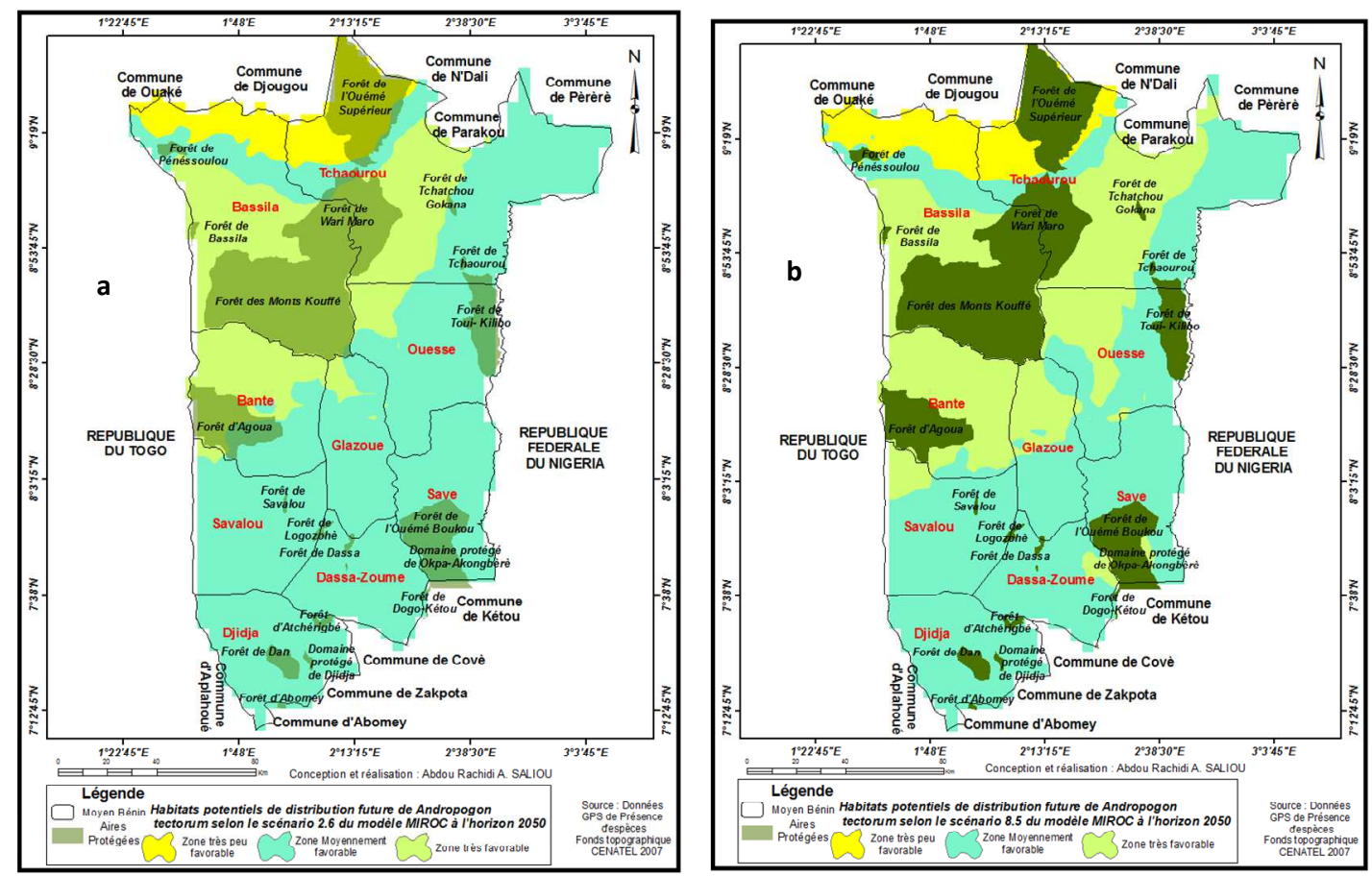

Figures 6 (a,b): Prédictions de distribution de Andropogon tectorum selon les scénarii 2.6 et 8.5 du modèle climatique MIROC à l'horizon 2050 dans le Moyen-Bénin.
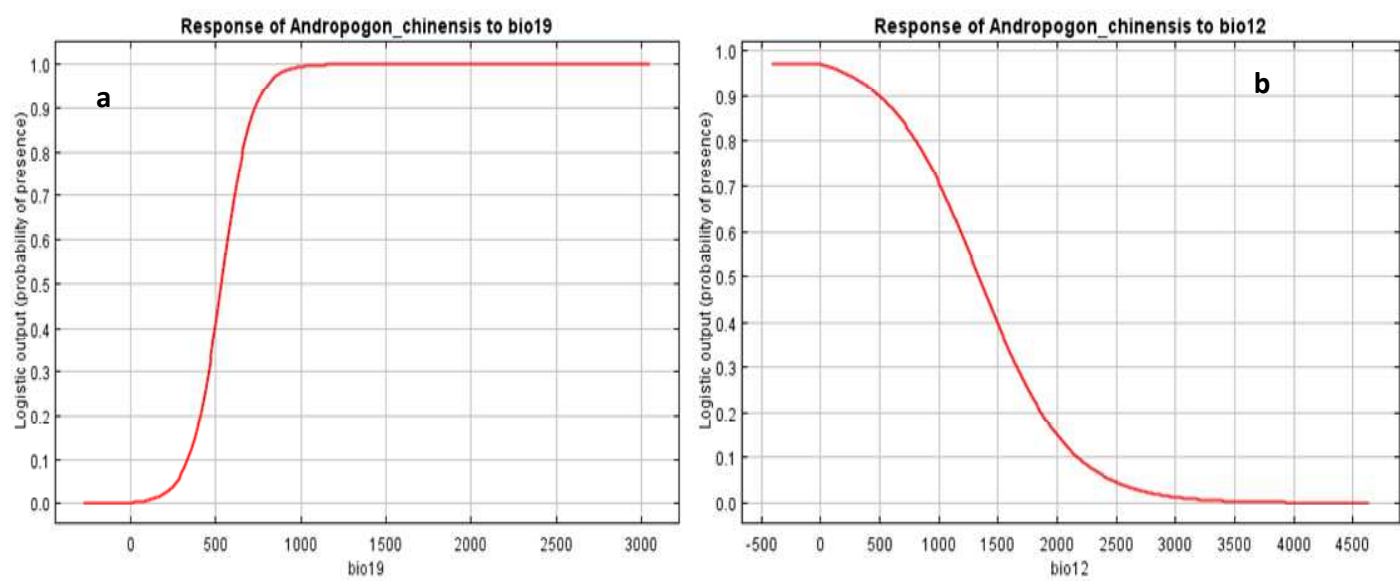

Figures 7: Réponses de Andropogon chinensis aux variables climatiques Bio19(a) et Bio12(b). 

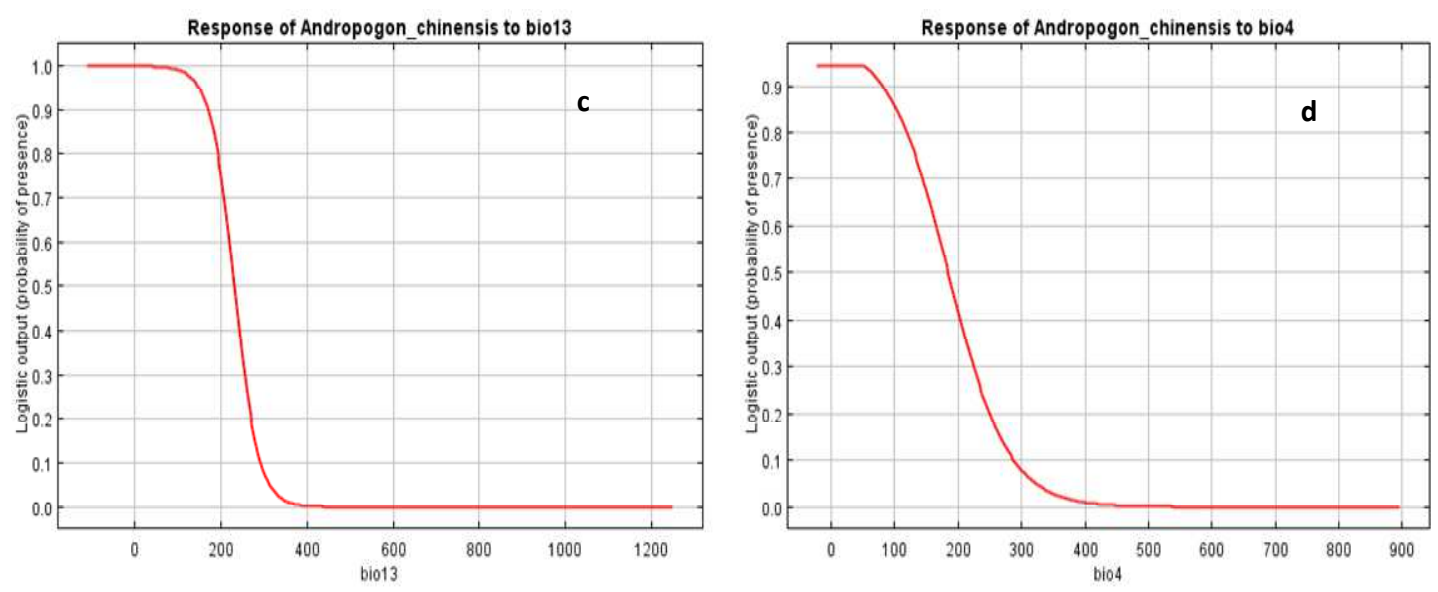

Figures 7: Réponses de Andropogon chinensis aux variables Bio13(c) et Bio4(d).
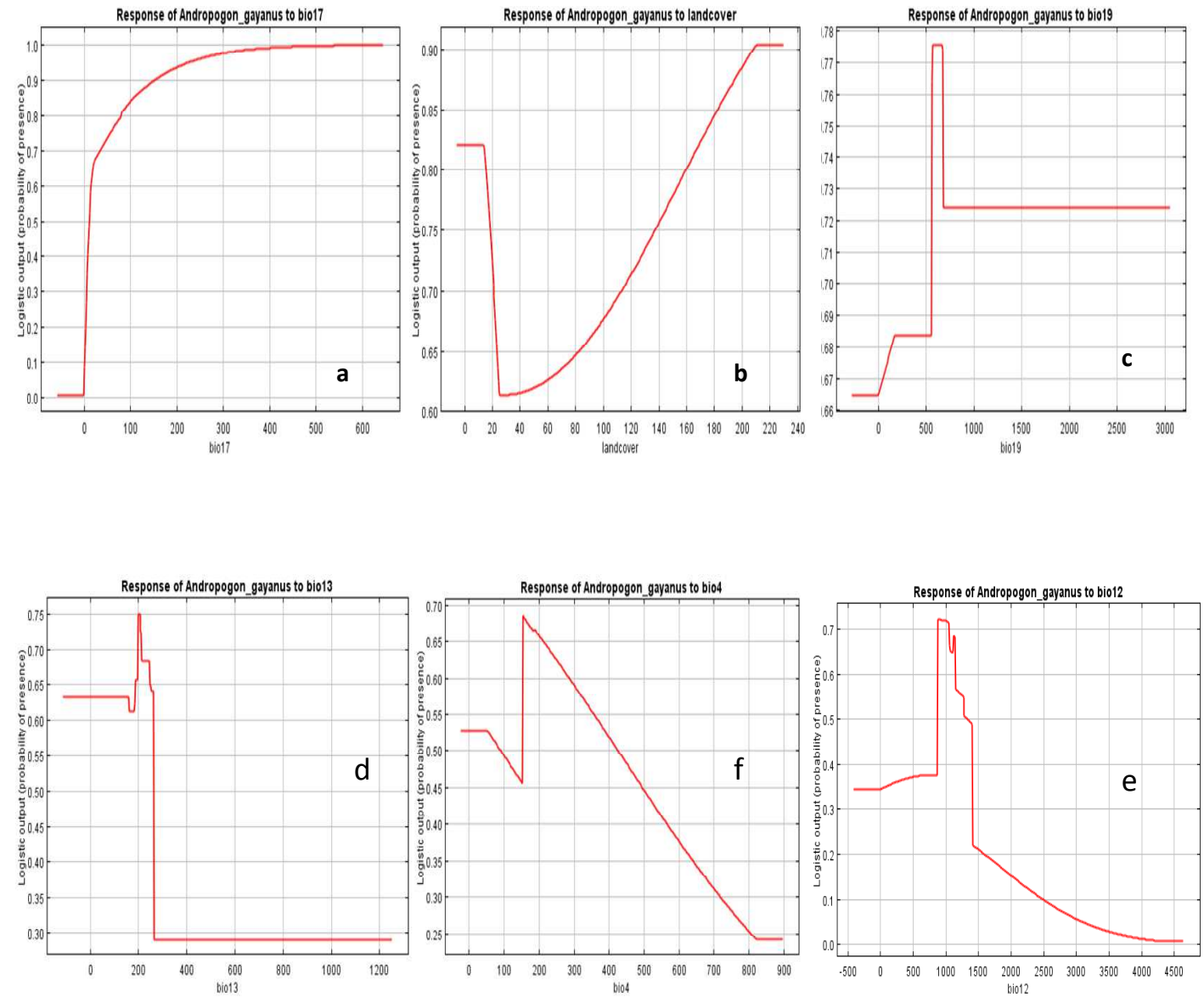

Figures 8: Réponses de Andropogon gayanus aux variables Bio17 (a), Landcover (b) et Bio19 (c), Bio13(d), Bio4 (e) et Bio12 (f). 
A. R. A. SALIOU et al. / Int. J. Biol. Chem. Sci. 8(6): 2696-2708, 2014
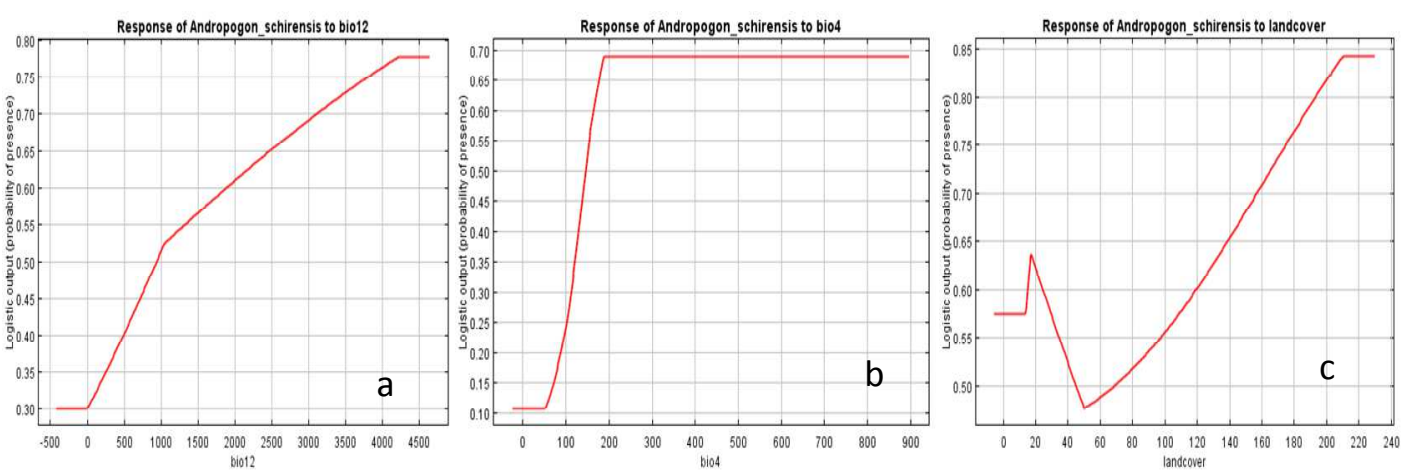

Figures 9 (a,b,c): Réponses de Andropogon schirensis aux variables.
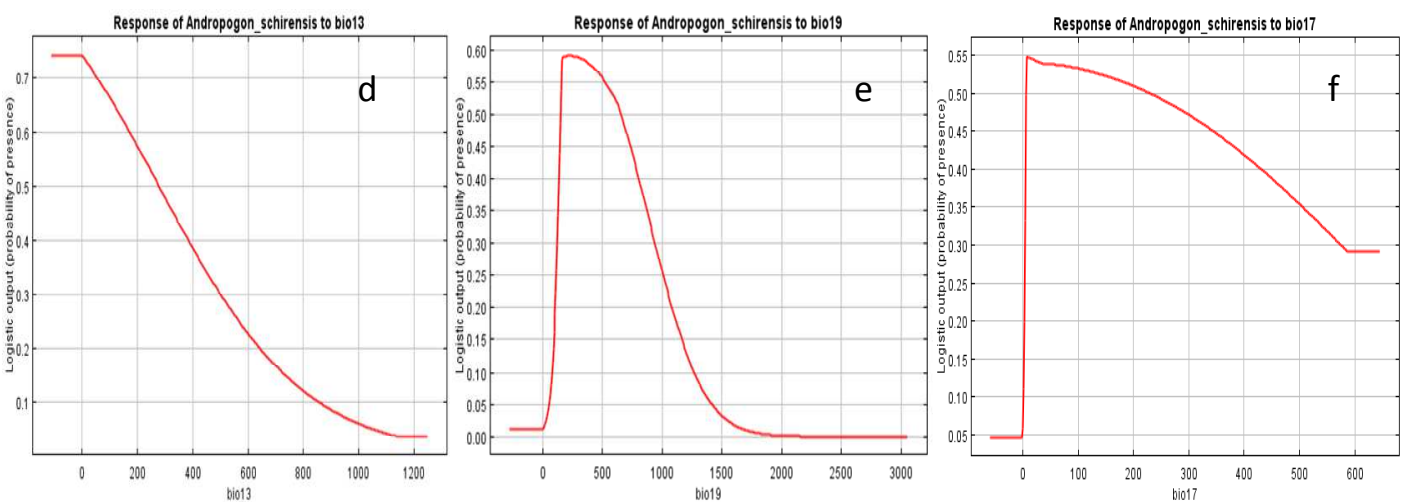

Figures 9: Réponses de Andropogon schirensis aux variables Bio12 (a), Bio4 (b) et Landcover (c), Bio13(d), Bio19(e) et Bio17(f).
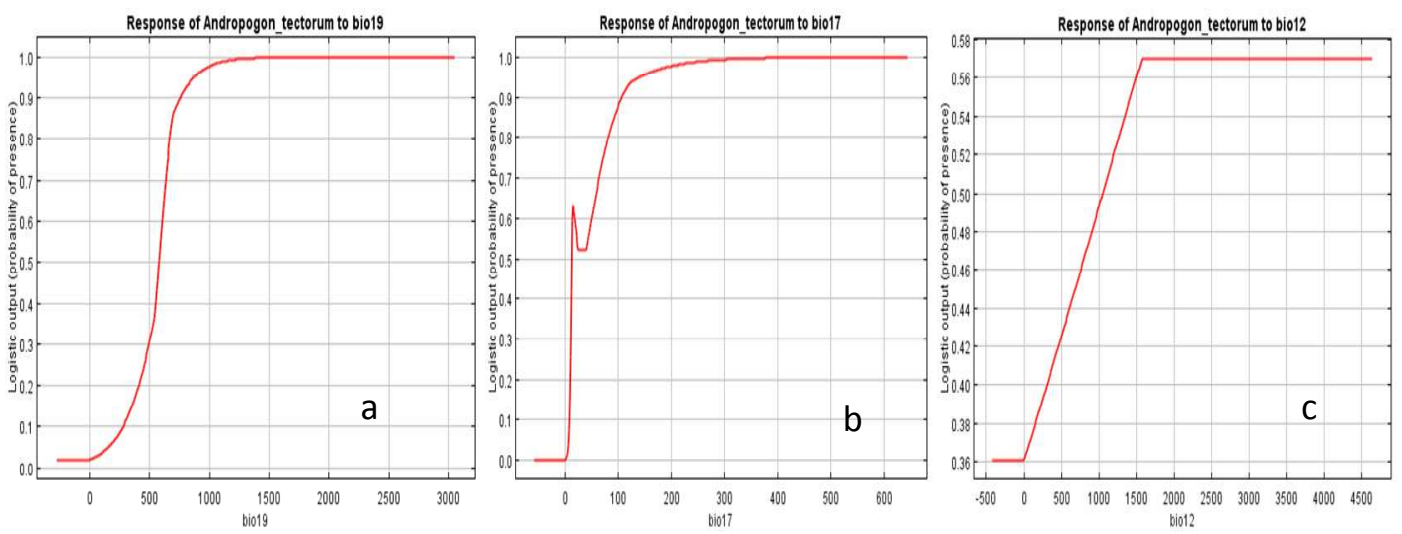

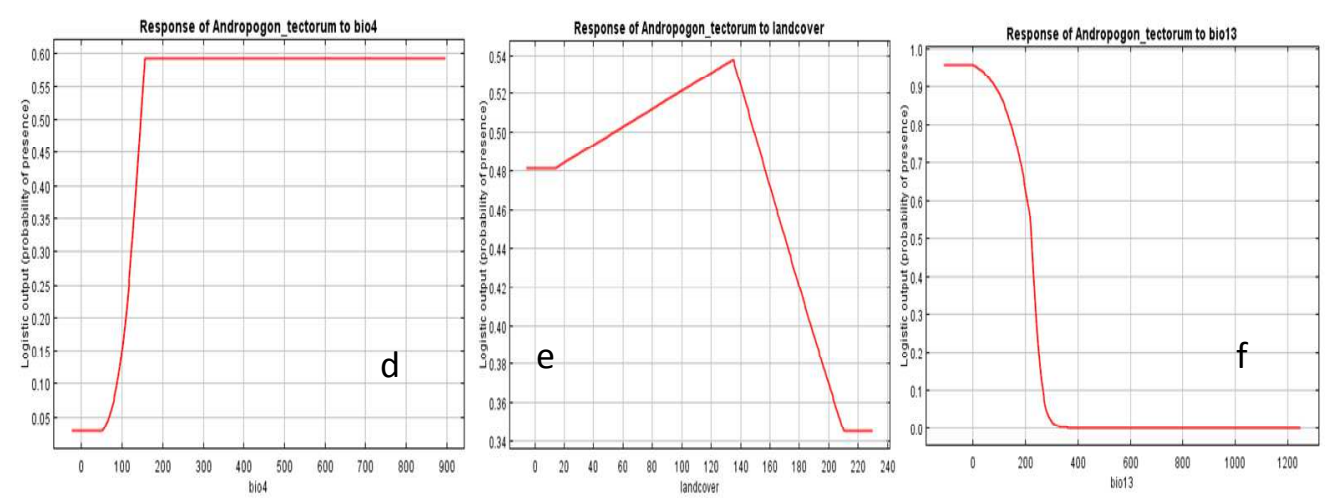

Figures 10: Réponses de Andropogon tectorum aux variables climatiques Bio19(a), Bio17(b) et Bio12(c), Bio4(d), Landcover(e) et Bio13(f).

\section{DISCUSSION}

Les deux scénarii du modèle MIROC utilisés prévoient des résultats différents en fonction des espèces pour les changements dans les précipitations d'ici 2050.

La prédiction de Andropogon chinensis montre quel que soit le scénario, une réduction de l'aire de distribution très favorable de l'espèce à l'horizon 2050 au profit des aires moyennement ou moins favorable. En effet, concernant RCP 2.6 pessimiste à la forte émission de Gaz à Effet de Serre avec une prédiction de l'augmentation des précipitations de 100 à $200 \mathrm{~mm}$ au Sud du pays, les aires de distribution très favorables dans le futur régresseront moins $(18,92 \%)$ que RCP 8.5 $(12,91 \%)$.

Par contre, tous les deux scénarii sans exception ont prédit une réduction des aires de distribution très favorables dans le futur au profit des aires moyennement ou moins favorables pour Andropogon gayanus. Ces aires très favorables passeront à l'horizon 2050 à $5,56 \%$ pour RCP 2.6 et $7,22 \%$ pour le scénario 8.5.

Quant à andropogon tectorum, tous les scénarii prédisent une augmentation de son aire de distribution très favorable à l'horizon 2050. Cette augmentation est prédite à $141,35 \%$ pour RCP 2.6 et $216,72 \%$ pour le scénario 8.5.
La prédiction à l'horizon 2050 de Andropogon schirensis montre une augmentation des aires très favorables pour les deux scénarii. Cette extension des aires de distribution est de $109,31 \%$ pour RCP 2.6 et $106,46 \%$ pour RCP 8.5.

La distribution à l'horizon 2050 de ces herbacées fourragères prédit une forte menace de Andropogon chinensis et de Andropogon gayanus aux variabilités climatiques liées à l'émission des Gaz à Effet de Serre (GES) dans le pays.

D'un autre point de vue, d'après Pulliam (2000) et Soberón (2007), les distributions des espèces ne sont pas uniquement contraintes par des facteurs abiotiques (ex. climat). Elles sont également façonnées par des interactions biotiques, des contraintes de dispersion, des effets anthropogéniques, des évènements stochastiques et autres facteurs historiques uniques et contingentes. Compte tenu du fait que la modélisation de la distribution potentielle de Andropogon schirensis, Andropogon gayanus, Andropogon chinensis, et Andropogon tectorum dans le Moyen-Bénin ait pris en compte les facteurs limitant les plus importants (température et précipitation) dans la distribution des espèces, du crédit peut être accordé à la qualité des résultats obtenus.

L'avantage de Maxent réside dans le fait qu'il ne nécessite en entrée que des 
données de présence, en complément des variables d'environnement (Peck et al., 2010). Il peut donc se passer de données d'absence pour fonctionner. Il permet aussi bien l'utilisation de variables quantitatives que qualitatives (Friedlaender et al., 2011). Des algorithmes déterministes efficients ont été développés et garantissent une convergence vers la distribution optimale (Elith et al., 2011).

Cependant, des critiques ont été porté sur le modèle Maxent. En effet, cette approche statistique parraît jeune, et n'est pas encore aussi éprouvée que d'autres modèles plus traditionnels, tels que les modèles linéaires généralisés ou les modèles additifs généralisés. En conséquence, il existe moins de ressources pour faciliter son utilisation (Phillips et al., 2006). Une trop forte auto corrélation spatiale dans les données d'observation peut introduire des biais dans les prédictions (Lahoz Monfort et al., 2010).

Bien que des critiques aient été soulevées à propos des modèles basées sur les données bioclimatiques à cause de leur exclusion des interactions biotiques et des scénarii de dispersion (Baker et al., 2000), ces modèles jouent un rôle vital dans l'évaluation des distributions potentielles des espèces (Pearson et Dawson, 2003).

Cependant, ces modèles ont aussi été très critiqués compte tenu de leurs faiblesses quant à prédire l'impact des variabilités climatiques sur la répartition géographique des espèces. Au nombre de ces faiblesses, on peut citer les incertitudes liées aux modèles utilisés, les difficultés à paramétrer les interactions écologiques, les réponses idiosyncratiques individuelles des espèces aux variabilités climatiques, les limitations de disséminations spécifiques à chaque espèce, la plasticité des limites physiologiques et les réponses adaptatives des agents disséminateurs (Elith et al., 2006 ; Schwartz, 2012).

\section{Conclusion}

La modélisation de la distribution des espèces est aujourd'hui facilitée par le développement de méthodes statistiques adaptées à différents types de situation et par la disponibilité croissante de bases de données environnementales. Les modèles prédictifs sont de plus en plus utilisés pour répondre aux questions relatives à l'écologie, à la biogéographie et à la conservation des espèces par l'identification des grands sites prioritaires. Ils peuvent également contribuer à la conservation des espèces en orientant des études biologiques vers des lieux où ces dernières sont susceptibles d'être retrouvées. Ces modèles pourraient en effet être utilisés afin d'évaluer l'adéquation des conditions environnementales d'un site avec la présence d'une espèce que l'on souhaiterait $\mathrm{y}$ implanter.

Dans cette étude, il a été développé un modèle de distribution géographique potentielle de Andropogon schirensis, Andropogon gayanus, Andropogon chinensis, et Andropogon tectorum dans le MoyenBénin.

\section{REMERCIEMENTS}

Nous remercions vivement l'Université d'Abomey-Calavi qui a financé cette étude, à travers le Programme du fonds compétitif $\mathrm{MDP} / 3 \mathrm{C}$, et les experts qui ont évalué cet article.

\section{REFERENCES}

Baker RH, Sansford CE, Jarvis CH, Cannon RJ, MacLeod A, Walters KFA. 2000. The role of climatic mapping in predicting the potential geographical distribution of non indigenous pests under current and future climates. Agriculture, Ecosystems \& Environment, 82:57-71.

Djenontin JA, 2005. Interactions Elevage Environnement: Adaptation des modes d'élevage des bovins à l'extension des espaces cultivés au Nord - Est du Bénin XX.

Elith J, Graham CH, Anderson RP, Dudík M, Ferrier S, Guisan A, Hijmans RJ,Huettmann F, Leathwick JR, Lehmann A, Li J, Lohmann LG, Loiselle BA, 
Manion G, Moritz C, Nakamura M, Nakazawa Y, Overton JM, Peterson AT, Phillips SJ, Richardson K, ScachettiPereira R, Schapire RE, Soberón J, Williams S, Wisz MS, Zimmermann NE. 2006. Novel methods improve prediction of species' distributions from occurrence data. Ecography, 29: 129-151.

Elith J, Phillips SJ, Hastie T, Dudík M, Chee YE, Yates CJ. 2011. A statistical explanation of MaxEnt for ecologists. Diversity and Distributions, 17(1): 43-57.

Friedlaender AS, Johnston DW, Fraser WR, Burns J, Patrick NH, Costa DP. 2011. Ecological niche modeling of sympatric krill predators around Marguerite Bay, Western Antarctic Peninsula. Deep Sea Research Part II. Topical Studies in Oceanography, 58(13-16): 1729-1740.

Houinato MRB, Sinsin B. 2000. La pression agro-pastorale sur la zone riveraine de la Réserve de la Biosphère de la Pendjari. Tropicultura, 18: 112-117.

Lahoz-Monfort JJ, Guillera-Arroita G, MilnerVGulland, EJ, Young, RP, Nicholson E. 2010. Satellite imagery as a single source of predictor variables for habitat suitability modelling: how Landsat can inform the conservation of a critically endangered lemur. Journal of Applied Ecology, 47(5): 1094-1102.

Oumorou M. 2003. Etudes écologiques, floristiques, phytogéographique et phytosociologique des inselbergs du Bénin. Thèse de doctorat du 3ème cycle. Faculté des Sciences, Université Libre de Bruxelles, Belgique, 1-101.

Parry ML, Canziani OF, Palutikof JP, van der Linden PJ, Hanson CE. 2007. Climate Change 2007: Impacts, Adaptation and Vulnerability. Contribution of working group II to the 4th assessment report of the Intergovernmental Panel on Climate Change, Cambridge University Press, 433467.

Pearson RG, Dawson TP. 2003. Predicting the impacts of climate change on the distribution of species: are bioclimatic envelope models useful? Global Ecology \& Biogeography, 12: 361-371.

Peck M, Thorn J, Mariscal A, Baird A, Tirira D, Kniveton D. 2010. Focusing Conservation Efforts for the Critically Endangered Brown-headed Spider Monkey (Ateles fusciceps) Using Remote Sensing, Modeling, and Playback Survey Methods. International Journal of Primatology, 32(1): 134-148.

Phillips SJ, Anderson RP, Schapire RE. 2006. Maximum entropy modeling of species geographic distributions. Ecological Modelling, 190: 231-259.

Pulliam H. 2000. On the relationship between niche and distribution. Ecology Letters, 3 (4): 349-361.

Reed K, Archer J, Peterson AT. 2008. Ecologic Niche Modeling of Blastomyces dermatitidis in Wisconsin. PLOS ONE 3(4): e2034. doi:10.1371/journal. pone.0002034.

Rodríguez JP, Brotons L, Bustamante J, Seoane J. 2007. The application of predictive modeling of species distribution to biodiversity conservation. Diversity \& Distribution DOI:0.1111/j.1472-4642.2007.00356.x.

Schwartz M, Caro T, Banda T. 2002. Assessing the sustainability of harvest of Pterocarpus angolensis in Rukwa Region, Tanzania. Forest Ecology and Management, 170: 259-269.

Soberón J. 2007. Grinnellian and Eltonian niches and geographic distribution of species. Ecology Letters, 10: 1115-1123.

Zekpete EIS, 2009. Transhumance et changement climatique: utilisation des outils d'aide à la décision dans la gestion durable des ressources des écosystèmes agropastoraux Soudano Sahéliens du Bénin DESS/AGRN/ FSA/UAC, 78p. 